\title{
Analysis of the Distribution of Erythrocyte Sodium Lithium Countertransport in a Sample Representative of the General Population
}

\author{
Eric Boerwinkle, Stephen T. Turner, Richard Weinshilboum, Mark Johnson, \\ Elliott Richelson, and Charles F. Sing
}

Department of Human Genetics (E.B., C.F.S.), University of Michigan, Ann Arbor; Division of Hypertension (S. T.T.) and Department of Pharmacology (R.W., M.J., E.R.), Mayo Clinic, Rochester, Minnesota

\begin{abstract}
Numerous studies of sodium-lithium countertransport (Na-Li CNT) have reported higher rates in essential hypertensives versus normotensive controls. We studied the distribution and the mode of inheritance of Na-Li CNT using a sample of 238 unrelated individuals and a sample of 245 individuals in 50 pedigrees all sampled from the population at large. The distribution of $\mathrm{Na}-\mathrm{Li} \mathrm{CNT}$ is continuous and bimodal. Our results indicate that there is a large genetic contribution to the distribution of Na-Li CNT. The hypothesis that the effect that causes bimodality is transmitted from generation to generation is supported by the fit to these data of a restricted transmission model with $\tau_{2}=0.749$. We hypothesize that this deviation of $\tau_{2}$ from its Mendelian expectation may be attributable to heterogeneity in the etiology of the bimodality in the Na-Li CNT distribution in the population at large.
\end{abstract}

Key words: complex segregation analysis, major gene, blood pressure, hypertension

\section{INTRODUCTION}

The search for biochemical phenotypes that relate genetic factors to blood pressure variability and hypertension spans many years [Wolstenholm and Cameron, 1954; Weinshilboum, 1979; Boerwinkle et al, 1984]. Recently, the phenotypes in-

Received for publication April 15, 1986; revision accepted May 5, 1986.

Address reprint requests to Dr. Charles F. Sing, Department of Human Genetics, University of Michigan, 4708 Medical Science II, Box 0618, Ann Arbor, MI 48109-0010.

1986 Alan R. Liss, Inc. 
volved in red blood cell sodium transport have been suggested as measures of an etiological mechanism for determining interindividual blood pressure variation [Swales, 1983; Blaustein, 1984]. In particular, numerous studies of the maximal in vitro rate of red blood cell sodium lithium countertransport $(\mathrm{Na}-\mathrm{Li} \mathrm{CNT}$ ) have reported higher rates in essential hypertensives compared with normotensive controls [for examples, see Canessa et al, 1980; Turner et al, 1986]. Turner et al [1985] have reported that the distribution of red blood cell $\mathrm{Na}-\mathrm{Li} \mathrm{CNT}$ in the population at large is continuous and bimodal. The observation that both red blood cell Na-Li countertransport and renal lithium clearance are altered in hypertensives [Weder, 1985] supports the hypothesis that individuals in the upper mode of the distribution have an altered pathway of membrane sodium transport that contributes to the development of hypertension.

Increased Na-Li CNT in normotensive first degree relatives of individuals with essential hypertension, but not in relatives of normotensive controls without a family history of essential hypertension [Canessa et al, 1980] suggests that there may be a contribution of genetic variation to the distribution of Na-Li CNT levels. In a sample of 434 individuals from ten hypertensive-prone pedigrees, Dadone et al [1984] reported that a model of mixed environmental factors and polygenic inheritance or a model of polygenic inheritance alone explained a significant fraction of Na-Li CNT variability. In preliminary analyses of data on nuclear families, we concluded that a mixed model including the major effect of a single locus and polygenic inheritance fit the data significantly better than a model of polygenic inheritance alone [Boerwinkle et al, 1984].

In this study we combine these family data with the data from our earlier study of randomly ascertained unrelated individuals [Turner et al, 1985] to examine the bimodality in this larger sample and to investigate alternative modes of transmission of the factor(s) responsible for the bimodality from parents to their offspring. Our results indicate that genetic differences among individuals play a large role in determining the distribution of $\mathrm{Na}-\mathrm{Li} \mathrm{CNT}$ in the population at large. The study reported here does not confirm that a single major gene is the most likely explanation for the determination of the two overlapping component distributions. We hypothesize and investigate the possibility that the distribution of $\mathrm{Na}-\mathrm{Li} \mathrm{CNT}$ in the general population has a heterogenous etiology.

\section{METHODS}

\section{Sample}

Data analyzed in this study were derived from two separate samples of individuals. All individuals were white and were free from reported acute or chronic illness. The first sample consisted of 245 individuals that are members of 53 nuclear families from 50 pedigrees ascertained from households having at least one child in the Rochester, Minnesota school system. This sample has been described in detail by Keith et al [1983]. Children less than 4 years of age were excluded from blood donation and were therefore not included in this sample. The size of the nuclear families ranged from three to nine individuals with an average of 2.9 children per family. Three of the families could be connected into one pedigree, two of the families could be connected into another, and the remaining families were assumed to be unrelated. 
To obtain more information about the distribution of Na-Li CNT in the population at large than was present in the sample of nuclear families, a second sample consisting of 238 unrelated individuals who donated blood at the Mayo Clinic blood bank was also included in the analysis of the causes of bimodality. A detailed description of this sample, the Na-Li CNT distribution, and the relationship between an individual's $\mathrm{Na}-\mathrm{Li} \mathrm{CNT}$ value and blood pressure were reported elsewhere [Turner et al, 1985].

\section{Sodium-Lithium Countertransport Assay}

Blood samples anticoagulated with heparin were assayed on the day of phlebotomy. Na-Li CNT was determined as the rate of sodium-dependent lithium efflux from lithium-loaded red blood cells using methods developed by Canessa and Tosteson [1979]. For loading with lithium, washed red cells were suspended at a hematocrit of approximately $20 \%$ in a solution consisting of $150 \mathrm{mM}$ lithium chloride, $10 \mathrm{mM}$ glucose, and $10 \mathrm{mM}$ Tris-MOPS, $\mathrm{pH}$ 7.4. This cell suspension was incubated in a shaking water bath for $3 \mathrm{hr}$ at $37^{\circ} \mathrm{C}$. After loading, the cells were washed four times in a washing solution consisting of $75 \mathrm{mM}$ magnesium chloride, $85 \mathrm{mM}$ mannitol, 10 $\mathrm{mM}$ glucose, and $10 \mathrm{mM}$ Tris-MOPS, $\mathrm{pH}=7.4$ at $40^{\circ} \mathrm{C}$, to remove external lithium, and a final suspension of washed red cells in washing solution (approximately $50 \%$ suspension) was prepared and kept at $40^{\circ} \mathrm{C}$ for the lithium efflux measurements.

To measure lithium efflux, the Li-loaded and washed red blood cells (RBC's) were incubated in parallel in solutions containing either $150 \mathrm{mM} \mathrm{NaCl}, 10 \mathrm{mM}$ glucose, $10 \mathrm{mM}$ Tris-MOPS $\mathrm{MgCl}_{2}, 85 \mathrm{mM}$ mannitol, $10 \mathrm{mM}$ glucose, $10 \mathrm{mM}$ TrisMOPS ( $\mathrm{pH}=7.4$ ), and $0.1 \mathrm{mM}$ ouabain (sodium medium) or $7.5 \mathrm{mM} \mathrm{MgCl} 2,85$ $\mathrm{mM}$ mannitol, $10 \mathrm{mM}$ glucose, $10 \mathrm{mM}$ Tris-MOPS $(\mathrm{pH}=7.4)$, and $0.1 \mathrm{mM}$ ouabain (magnesium medium). The transport assay was started by pipetting $0.15 \mathrm{ml}$ of RBC suspension into nine microfuge tubes, each containing 1.35 of magnesium medium. The tubes were then placed in a shaking water bath at $37^{\circ} \mathrm{C}$. At 10,20 , and $30 \mathrm{~min}$, three tubes containing sodium medium and three tubes containing magnesium medium were removed from the water bath, cooled quickly to $4{ }^{\circ} \mathrm{C}$, and centrifuged. Supernatant was quickly pipetted off the cell pellet and saved for triplicate determinations of lithium concentration in the two types of media.

Lithium concentrations in the efflux media were determined by atomic emission spectrophotometry at a wavelength of $670.8 \mathrm{~nm}$. Lithium concentrations in the samples containing sodium media were read against standard solutions of lithium chloride. The lithium content of each efflux sample was expressed per volume of $\mathrm{RBC}$ in the sample, and the triplicate determinations at each time point were averaged. The Li efflux rates in the sodium and magnesium media (expressed as mmol of lithium/liter of $\mathrm{RBC} / \mathrm{h}=\mathrm{mmol} / \mathrm{L} \mathrm{RBC} / \mathrm{hr}$ ) were taken as the slopes of the linear regression lines calculated with $\mathrm{Li}$ efflux (mmol/L $\mathrm{RBC}$ ) as a function of time (hr). The relationship between $\mathrm{Li}$ efflux and time was linear in both sodium and magnesium media. The data were considered acceptable only when the regression lines explained greater than $95 \%$ of the variation in $\mathrm{Li}$ efflux. The difference between the slope of the regression lines for sodium and magnesium samples was the measure of $\mathrm{Na}-\mathrm{Li}$ CNT.

Prior to this study, the reliability of the Na-Li CNT assay in our laboratory was tested. Duplicate blood samples were drawn from five healthy individuals and analyzed on the same day. The standard deviation among repeated assays of the same 
sample was $0.20 \mathrm{mmol} / \mathrm{L} \mathrm{RBC/hr}$ for a coefficient of variation equal to $7.63 \%$ of the mean Na-Li CNT value. The stability over time of Na-Li CNT was assessed by three to five measurements over a 3-month period in the same five individuals. The coefficient of variation among measurements within individuals averaged $13.0 \pm$ $2.8 \%$ in these subjects. These levels of technical error and intraindividual variation in Na-Li CNT are similar to those reported by others [Trevisan et al, 1981].

\section{Statistical Analyses}

Prior to commingling and genetic analyses, the Na-Li CNT values for the sample of unrelated blood bank donors were adjusted so that their mean was equal to the mean of the sample of nuclear families. Box's F test [Box, 1949] was used to test the hypothesis that the variances were homogeneous between the samples. A maximum likelihood method [Day, 1969; Boerwinkle et al, 1986] was employed to determine whether the distribution of $\mathrm{Na}-\mathrm{Li} \mathrm{CNT}$ in these data was explained by multiple commingled normal distributions. A model with a mixture of two normal distributions with equal variances was compared to a model having one normal distribution. Previous analyses carried out separately on the blood bank donors and the sample of families indicated that a model with three distributions does not fit the observed Na-Li CNT distribution better than a model with two distributions [Boerwinkle et al, 1984; Turner et al, 1985]. The parameters of the two-component model include the means of each component $\left(\mu_{1}, \mu_{2}\right)$, the relative frequency of first component $\left(f_{1}\right)$, and the within-component variance $\left(\sigma^{2}\right)$. A test of the null hypothesis of one component distribution was carried out by restricting parameters of the two components model to hypothesized values. The difference between the maximum values of the $\log _{e}$ likelihood under the two-component and one-component models forms a basis for judging competing hypotheses. This difference multiplied by -2 is distributed approximately as a chi-square distribution with degrees of freedom equal to the number of parameters restricted by the hypothesis.

Using multiple linear regression, we estimated the extent to which variability in $\mathrm{Na}-\mathrm{Li}$ CNT was attributable to generational differences where appropriate (ie, parents and children), gender differences, and age variability within generations. To reduce residual skewness in the data adjusted for these concomitants, we applied the power transformation suggested by MacLean et al [1976] for a model of multiple commingled distributions. This transformation is given by the following:

$$
y=6 / P\left[(x / 6+1)^{P}-1\right]
$$

In this transformation, $\mathrm{x}$ is the untransformed $\mathrm{Na}-\mathrm{Li} \mathrm{CNT}$ value, $\mathrm{P}$ is the skewness parameter, and $\mathrm{y}$ is the transformed $\mathrm{Na}-\mathrm{Li} \mathrm{CNT}$ value.

The mode of transmission of Na-Li CNT from parents to offspring was investigated using complex segregation analysis [Elston and Stewart, 1971; Lalouel et al, 1983]. We assumed that the distribution of the phenotype is a consequence of the independent contributions of either a genetic or an environmental major effect that determines bimodality, polygenic effects, and nongenetic individual specific effects that determine differences among individuals within components. The major effect was modeled as two alternative factors, $A_{1}$ and $A_{2}$, that may be of either genetic or environmental origin. These two factors combine to define three classes, or ousiotypes [Cannings et al, 1978], of individuals, $A_{1} A_{1}, A_{1} A_{2}$, and $A_{2} A_{2}$. The relative 
frequency of the $A_{1}$ factor is denoted $p$ and the relative frequency of the $A_{2}$ factor, $q$, is equal to $1-P$. Assuming Hardy-Weinberg equilibrium, the relative frequencies of these three types are equal to $f\left(A_{1} A_{1}\right)=p^{2}, f\left(A_{1} A_{2}\right)=2 p q$, and $f\left(A_{2} A_{2}\right)=q^{2}$.

We let $\tau_{1}, \tau_{2}$, and $\tau_{3}$ be the probabilities that ousiotypes $\mathrm{A}_{1} \mathrm{~A}_{1}, \mathrm{~A}_{1} \mathrm{~A}_{2}$, and $\mathrm{A}_{2} \mathrm{~A}_{2}$ transmit the $A_{1}$ factor to their children, respectively. For the general transmission model, these transmission probabilities were each estimated under the constraint that they be between 0 and 1 . The major gene model defines the segregation of two factors, alleles, at a single autosomal locus. The probabilities of transmitting alleles from their parents to their offspring are assumed to be determined by Mendelian principles. The Mendelian transmission probabilities are as follows: $\tau_{1}=1.0, \tau_{2}=$ 0.5 , and $\tau_{3}=0.0 . \tau_{2}=0.5$ is the sine qua non of Mendelian transmission. It has been suggested [Lalouel et al, 1983] that one should consider a test of $\tau_{2}=0.5$ before accepting a major gene model, although tests based on $\tau_{2}$ alone may not provide enough safeguard against falsely inferring a major gene [Demenais et al, 1986]. A model with $\tau_{1}$ and $\tau_{3}$ fixed at their Mendelian transmission values while allowing $\tau_{2}$ to be unconstrained was fit to these data for this purpose. We term this model a restricted transmission model. The major environmental effect model is assumed to determine bimodality of the phenotype in both parents and children but is not transmitted from generation to generation. Each of the transmission probabilities for this model were taken to be equal to the frequency of the $A_{1}$ factor (p). This major environmental effect model dictates that the probability that an individual is of one major environmental type or the other is independent of their generation or the ousiotype of their parents. The transmission probabilities under both the genetic and the environmental major effects models considered here generate an equilibrium frequency distribution of the ousiotypes from generation to generation.

Both major effects models assume that the distribution of Na-Li CNT among individuals having the same ousiotype is normal with variance, $\sigma^{2}$. A fraction of this variance component is attributable to variability in the additive effects of polygenic and environmentally transmitted factors that are subsumed by the parameter that defines a polygenic mode of inheritance $\left(h^{2}\right)$, and a fraction $\left(1-h^{2}\right)$ is attributable to residual variability that includes individual specific environmental effects and measurement error. The likelihood that a given model explains the distribution of $\mathrm{Na}$ Li CNT among the pool sample of unrelated blood bank donors and family members was computed using PAP [Hasstedt and Cartwright, 1981]. The numerical approximation to the exact likelihood given by Hasstedt [1982] and the numerical optimization methods given by Lalouel [1979] were used to obtain the maximum likelihood estimates of parameters associated with each of the models that were considered. Hypothesis testing was carried out by using the chi-square $\left(\chi^{2}\right)$ approximation to minus twice the $\log _{e}$ of the ratio of the maximum likelihood of a complete model to the maximum likelihood of a reduced model with one or more of the parameters constrained to a hypothesized value. Hypothesis testing was carried out in a hierarchical manner by applying the principles discussed by Lalouel et al [1983]. In the first stage, we compared the general model first with a model with no major effect and then with the major environmental effects model. Rejection of these two models was followed by a second stage to determine which transmission model fit these data best. We compared the general transmission model with the restricted transmission model where $\tau_{1}=1.0, \tau_{3}=0.0$, and $\tau_{2}$ was estimated. This restricted transmission model was then compared to the Mendelian transmission model. 


\section{RESULTS \\ Commingling Analyses}

Descriptive statistics for the blood bank donors and the family members available for this study are presented in Table I. The ages ranged from 4 to 67 years old. The average age of the adults (blood bank donors and parents) and children in the pedigrees was 37.39 years (range $=18-67$ ) and 14.15 years (range $=4-28$ ), respectively. In the pooled sample of 483 individuals, there were 271 males and 212 females. The average Na-Li CNT value was higher in the nuclear families than in the sample of blood bank donors $(0.341$ vs 0.289$)$, higher in males than in females $(0.335$ vs 0.290$)$, and higher in parents than in their children $(0.358$ vs 0.328$)$. These effects of gender and age are consistent with other reports in the literature [reviewed by Turner et al, 1986].

Commingling analyses (Table II) were done separately for these two samples prior to the pooling of the data to assure that the bimodality within each sample was homogenous between samples. An analysis of multimodality on the observed $\mathrm{Na}-\mathrm{Li}$ CNT values rejected the hypothesis of a single normal distribution in favor of two normal distributions for each sample (for the blood bank donors, $\chi^{2}=19.43,2 \mathrm{df}, \mathrm{P}$ $<.0001$; for the families, $\left.\chi^{2}=20.05,2 \mathrm{df}, \mathrm{P}<.0001\right)$. Maximum likelihood estimates of the two means, the relative frequency of the lower component distribution, and the standard deviation that describes the dispersion within each component are given in Table II for each sample. The parameters estimated for the two models for each of the two samples are very similar. An analysis of the pooled sample predicts that $75 \%$ of the population from which these samples were drawn belongs to the first distribution with a mean Na-Li CNT value of $0.293 \mathrm{mmol} / \mathrm{L} \mathrm{RBC} / \mathrm{hr}$, and

TABLE I. Mean (Standard Deviation) for Age and Observed Na-Li CNT Values for Each Sample, by Generation and Gender

\begin{tabular}{|c|c|c|c|c|c|}
\hline Sample & Generation & Sex & No. & Age (years) & $\begin{array}{c}\text { Na-Li CNT } \\
\mathrm{mmol} / \mathrm{L} \mathrm{RBC} / \mathrm{hr}\end{array}$ \\
\hline \multirow[t]{2}{*}{ Blood bank donors } & $\mathrm{NA}^{\mathrm{a}}$ & Male & 144 & $\begin{array}{c}36.96 \\
(10.22)\end{array}$ & $\begin{array}{c}0.305 \\
(0.120)\end{array}$ \\
\hline & & Female & 94 & $\begin{array}{c}33.43 \\
(10.09)\end{array}$ & $\begin{array}{c}0.265 \\
(0.108)\end{array}$ \\
\hline \multirow[t]{4}{*}{ Family } & Parents & Male & 54 & $\begin{array}{l}42.40 \\
(5.04)\end{array}$ & $\begin{array}{c}0.403 \\
(0.135)\end{array}$ \\
\hline & & Female & 48 & $\begin{array}{c}40.54 \\
(5.89)\end{array}$ & $\begin{array}{c}0.307 \\
(0.108)\end{array}$ \\
\hline & Children & Male & 73 & $\begin{array}{l}14.57 \\
(4.08)\end{array}$ & $\begin{array}{c}0.344 \\
(0.103)\end{array}$ \\
\hline & & Female & 70 & $\begin{array}{l}13.70 \\
(3.49)\end{array}$ & $\begin{array}{c}0.312 \\
(0.076)\end{array}$ \\
\hline \multirow[t]{3}{*}{ Pooled } & $\mathrm{NA}^{\mathbf{a}}$ & Male & 271 & $\begin{array}{c}31.66 \\
(12.32)\end{array}$ & $\begin{array}{c}0.335 \\
(0.121)\end{array}$ \\
\hline & & Female & 212 & $\begin{array}{c}28.45 \\
(12.40)\end{array}$ & $\begin{array}{c}0.290 \\
(0.098)\end{array}$ \\
\hline & & Pooled & 483 & $\begin{array}{c}30.24 \\
(12.40)\end{array}$ & $\begin{array}{c}0.316 \\
(0.113)\end{array}$ \\
\hline
\end{tabular}

${ }^{a} \mathrm{NA}$, not applicable. 


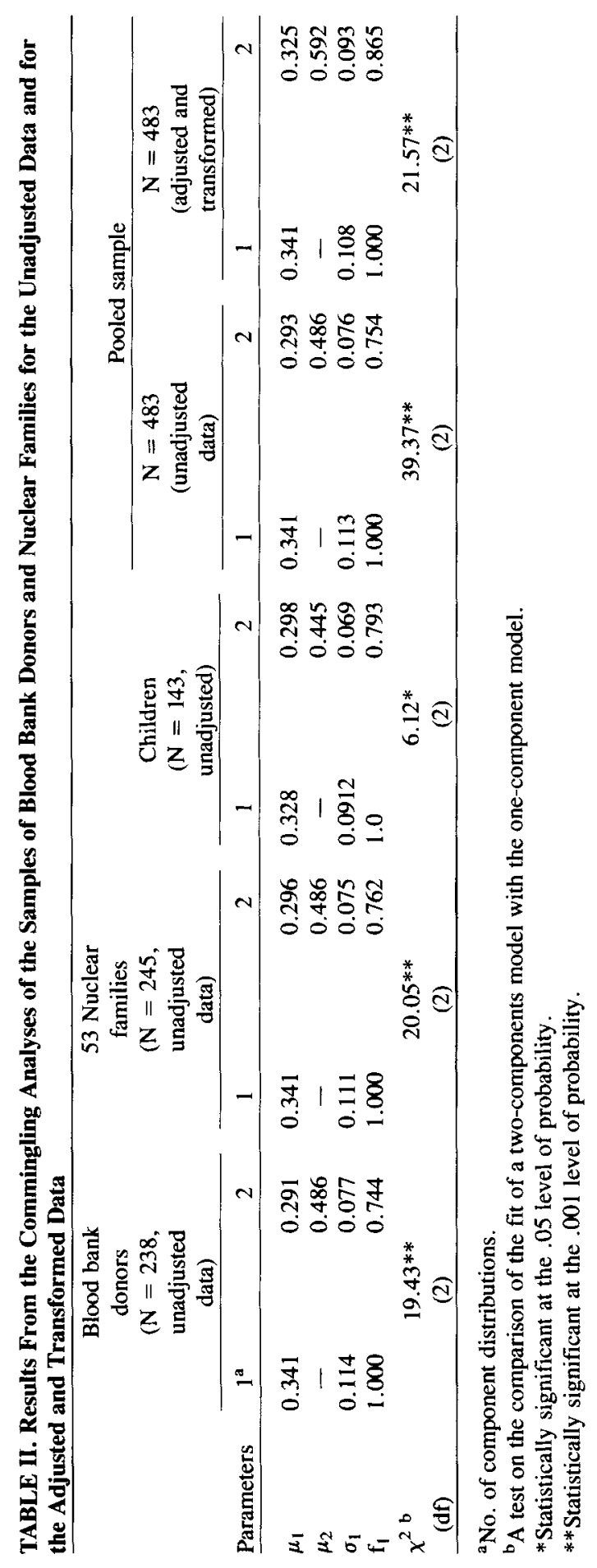


$25 \%$ of the population belongs to the second distribution, with a higher mean value of $0.486 \mathrm{mmol} / \mathrm{L} \mathrm{RBC} / \mathrm{hr}$.

The bimodality estimated for the raw data could be due to differences in $\mathrm{Na}-\mathrm{Li}$ CNT values attributable to variability of the concomitants and/or due to skewness. The distribution of Na-Li CNT in both samples is significantly skewed toward higher values $\left(\mathrm{g}_{1}=0.55\right.$ for the blood bank donors, and $\mathrm{g}_{1}=0.53$ for the nuclear families, $\mathrm{P}<.02$ in each case). To examine these effects, commingling analyses were carried out on the data after adjusting for the effects of concomitant variability (generational differences, gender and age variability within generations) and after a power transformation was carried out to reduce residual skewness. The difference between the average male and female $\mathrm{Na}-\mathrm{Li} \mathrm{CNT}$ values accounted for only $5 \%$ of the total variation. Generational differences and age variability within generations accounted for an additional 3\%. The $\mathrm{Na}-\mathrm{Li}$ CNT values within each sex-sample strata were adjusted to have the mean of the unadjusted Na-Li CNT valve of the sample of nuclear families (ie, 0.341 ). The standard deviation of the pooled sample of 483 individuals decreased from 0.113 to 0.108 when the data were adjusted for concomitant variability. The maximum likelihood estimate of the power in equation 1 that minimized residual skewness in the adjusted data was equal to 1.24. A model with one normal distribution was rejected in favor of a model with two normal distributions for the adjusted and transformed data $\left(\chi^{2}=21.57,2 \mathrm{df}, \mathrm{P}<.0001\right)$. Parameter estimates for these models are also given in Table II. The adjusted and transformed data predicts that $86.5 \%$ of the population belongs to a distribution with a mean of $0.325 \mathrm{mmol} / \mathrm{L} \mathrm{RBC} / \mathrm{hr}$, and $13.5 \%$ of the population belongs to the second distribution with a mean of $0.592 \mathrm{mmol} / \mathrm{L} \mathrm{RBC} / \mathrm{hr}$. This result suggests that it is unlikely that variability in the concomitants and/or skewness in the data accounts for the observed bimodality of the distribution of Na-Li CNT.

Both of the models considered by the segregation analysis presented below (ie, the major genetic effect model and the major environmental effect model) dictate that the phenotypic distribution in the children of the families also fits a mixture of two normal distributions. Hence, we also carried out a separate commingling analysis on the subsample of 143 children from the sample of nuclear families. This analysis also rejected the hypothesis of a single normal distribution in favor of a hypothesis of two normal distributions $\left(\chi^{2}=6.12,2 \mathrm{df}, \mathrm{P}<.05\right)$. The maximum likelihood estimates of the parameters of this model were very similar to the estimates from the pooled sample of blood bank donors and families given in Table II. This result indicates that the underlying factor that is contributing to the bimodality is also present in children.

\section{Genetic Analyses of Na-Li CNT}

Evidence for familial aggregation of both unadjusted and adjusted and transformed Na-Li CNT values is given in Table III. That the observed correlations are likely due to shared genes rather than shared environments is reflected in a larger correlation between parents and offspring and between sets of full sibs than between spouses.

We then applied complex segregation analysis to examine the distribution of observed Na-Li CNT values among the 238 unrelated blood bank donors and 245 individuals from 53 nuclear families. A comparison of the fit of selected models to the unadjusted data is presented in Table IV. Because these data cannot distinguish between dominant and recessive general transmission models, investigation of other 
TABLE III. The Pearson Product Moment Correlations Between Spouses, Between the Midparental Value and a Randomly

Selected Offspring, and the Sibling Correlation Estimated by the Weighted Average (Across Sibship Sizes) of the Intraclass

Correlation Between Full Siblings

\begin{tabular}{lccc}
\hline & Spouses & $\begin{array}{c}\text { Midparent- } \\
\text { offspring }\end{array}$ & Siblings \\
\hline Unadjusted & -0.088 & 0.302 & 0.379 \\
$\begin{array}{l}\text { Adjusted and } \\
\text { transformed }\end{array}$ & -0.095 & 0.304 & 0.342 \\
\hline
\end{tabular}

TABLE IV. Parameter Estimates and Tests of Hypotheses About the Distribution of Observed NaLi CNT Values Among 238 Unrelated Blood Bank Donors and 245 Individuals From 53 Nuclear Families

\begin{tabular}{|c|c|c|c|c|c|}
\hline Parameters & $\begin{array}{c}\text { General } \\
\text { transmission } \\
\end{array}$ & $\begin{array}{c}\text { No } \\
\text { major } \\
\text { effect }\end{array}$ & $\begin{array}{c}\text { Major } \\
\text { environmental } \\
\text { effect }\end{array}$ & $\begin{array}{c}\text { Restricted } \\
\text { transmission }\end{array}$ & $\begin{array}{c}\text { Major } \\
\text { genetic } \\
\text { effect }\end{array}$ \\
\hline \multicolumn{6}{|c|}{$\begin{array}{l}\text { A. Recessive mode of inheritance } \\
\text { of elevated levels }\end{array}$} \\
\hline$\mu_{1}$ & 0.294 & 0.341 & 0.296 & 0.294 & 0.293 \\
\hline$\mu_{2}$ & 0.294 & 0.341 & 0.296 & 0.294 & 0.293 \\
\hline$\mu_{3}$ & 0.486 & 0.341 & 0.483 & 0.486 & 0.483 \\
\hline $\mathrm{p}$ & 0.475 & 1.000 & 0.519 & 0.482 & 0.493 \\
\hline$\sigma$ & 0.078 & 0.113 & 0.079 & 0.075 & 0.077 \\
\hline$h^{2}$ & 0.494 & 0.509 & 0.519 & 0.332 & 0.290 \\
\hline$\tau_{1}$ & 0.478 & - & 0.519 & 1.000 & 1.000 \\
\hline$\tau_{2}$ & 0.893 & - & 0.519 & 0.749 & 0.500 \\
\hline$\tau_{3}$ & 0.514 & - & 0.519 & 0.000 & 0.000 \\
\hline $\begin{array}{l}\chi^{2} \text { difference } \\
\text { from general } \\
\text { transmission } \\
\text { model (df) }\end{array}$ & - & $\begin{array}{c}47.0 * * * \\
(5)\end{array}$ & $\begin{array}{l}14.2^{* *} \\
(3)\end{array}$ & $\begin{array}{c}4.2 \mathrm{NS}^{\mathrm{a}} \\
(2)\end{array}$ & $\begin{array}{r}10.6^{*} \\
(3)\end{array}$ \\
\hline \multicolumn{6}{|c|}{ B. Dominant mode of inheritance of elevated levels } \\
\hline$\mu_{1}$ & 0.294 & 0.341 & 0.296 & 0.293 & 0.290 \\
\hline$\mu_{2}$ & 0.487 & 0.341 & 0.483 & 0.486 & 0.477 \\
\hline$\mu_{3}$ & 0.487 & 0.341 & 0.483 & 0.486 & 0.477 \\
\hline $\mathrm{p}$ & 0.850 & 1.000 & 0.877 & 0.847 & 0.849 \\
\hline$\sigma$ & 0.078 & 0.113 & 0.079 & 0.077 & 0.077 \\
\hline$h^{2}$ & 0.467 & 0.509 & 0.551 & 0.407 & 0.235 \\
\hline$\tau_{1}$ & 0.986 & - & 0.877 & 1.0 & 1.0 \\
\hline$\tau_{2}$ & 0.915 & - & 0.877 & 0.869 & 0.5 \\
\hline$\tau_{3}$ & 0.0 & - & 0.877 & 0.0 & 0.0 \\
\hline $\begin{array}{l}\chi^{2} \text { difference } \\
\text { from general } \\
\text { transmission } \\
\text { model }(\mathrm{df})\end{array}$ & - & $\begin{array}{c}51.2 * * * \\
(5)\end{array}$ & $\begin{array}{c}18.4 * * * \\
(3)\end{array}$ & $\begin{array}{c}0.0 \mathrm{NS}^{\mathrm{a}} \\
(2)\end{array}$ & $\begin{array}{c}18.0^{* * * *} \\
(3)\end{array}$ \\
\hline
\end{tabular}

${ }^{a} \mathrm{NS}$, not significant.

* Statistically significant at the .05 level of probability.

** Statistically significant at the .01 level of probability.

$* * *$ Statistically significant at the .001 level of probability. 
transmission models was done separately for each case. Using a model of Mendelian transmission, we found that a model of codominant inheritance did not fit the data significantly better than either a model of recessive or of dominance inheritance (data not shown). A model that does not include the effects of any major factor fit significantly worse $\left(\chi^{2}=47.0,5 \mathrm{df}, \mathrm{P}<.001\right.$ against the recessive general transmission model; 51.2, $5 \mathrm{df}, \mathrm{P}<.001$ against the dominant general transmission model) than the general transmission model in both the dominant and recessive cases. The maximum likelihood estimates of the two means of the bimodal Na-Li CNT distribution from the pedigree analysis are very similar to those given by the commingling analysis presented in Table II.

The general transmission model with a recessive allele for elevated values (A) fit the data significantly better than the major environmental factor model $(\mathrm{P}<.01)$. We next compared the general transmission model to models that examine specific types of transmission of the major factor from generation to generation. The restricted model with $\tau_{1}=1.0$ and $\tau_{3}=0.0$ fits as well as the general transmission model. The maximum likelihood estimate of $\tau_{2}$ for this model was 0.749 . The major gene model did not fit the data as well as either the general transmission model or the model estimates $\tau_{2}$. For the dominant case (B), the inferences are basically the same as for the recessive case. The major environmental effect model was also rejected at the .01 level of probability. The restricted model fit these data as well as the general transmission model, and the major gene mixed model did not explain these data as well as either the general transmission model or the restricted model. The model that restricts $\tau_{1}$ and $\tau_{3}$ at their Mendelian values and estimates $\tau_{2}$ gave the most satisfactory fit to these observed $\mathrm{Na}-\mathrm{Li} \mathrm{CNT}$ values with the fewest parameters. For both the dominant and recessive cases, a restricted transmission model without a polygenic component did not fit the data as well as the restricted transmission model with polygenes (data not shown, $\mathrm{P}<.05$ in both cases). The estimates of the contribution of polygenes given by the general transmission model and the major environmental effects model were similar. When the transmission probabilities were constrained to either the restricted transmission model or the Mendelian transmission model, the estimate of the polygenic contribution decreased. The estimates of the within-component variance were similar among all models that included parameters for bimodality.

We also asked if the inferences from our analyses of the observed Na-Li CNT values were similar for the adjusted and power transformed data. The adjusted and transformed data were not completely reanalysed but rather were used as a check of consistency with our results from the analyses of the raw data. Analyses were carried out for both dominant and recessive cases. Results from these analyses are given in Table V. For these adjusted and transformed data, the restricted transmission model fit the data as well as the general transmission model and significantly better than a model without the effect of a major factor $\left(\chi^{2}=17.4,3 \mathrm{df}, \mathrm{P}<.001\right.$ for the recessive case; $\chi^{2}=24.0,3 \mathrm{df}, \mathrm{P}<.001$ for the dominant case).

\section{DISCUSSION}

The results from our analyses of the samples of 238 blood bank donors and 50 pedigrees consisting of 245 individuals indicates that the frequency distribution of $\mathrm{Na}-\mathrm{Li} \mathrm{CNT}$ in the general population is continuous and bimodal. Bimodality is suggested from studies comparing normotensive and hypertensive individuals and has 
TABLE V. Parameter Estimates and Tests of Hypotheses About the Distribution of Adjusted and Transformed Na-Li CNT Values Among 238 Unrelated Blood Bank Donors and 245 Individuals From 53 Nuclear Families

\begin{tabular}{|c|c|c|c|c|c|}
\hline \multirow[b]{2}{*}{ Parameters } & \multicolumn{2}{|c|}{$\begin{array}{c}\text { Recessive } \\
\text { transmission }\end{array}$} & \multirow{2}{*}{$\begin{array}{c}\text { No } \\
\text { major } \\
\text { effect }\end{array}$} & \multicolumn{2}{|c|}{$\begin{array}{c}\text { Dominant } \\
\text { transmission }\end{array}$} \\
\hline & General & Restricted & & General & Restricted \\
\hline$\mu_{1}$ & 0.308 & 0.311 & 0.343 & 0.308 & 0.309 \\
\hline$\mu_{2}$ & 0.308 & 0.311 & 0.343 & 0.484 & 0.487 \\
\hline$\mu_{3}$ & 0.481 & 0.484 & 0.343 & 0.484 & 0.487 \\
\hline $\mathrm{p}$ & 0.527 & 0.549 & 1.0 & 0.880 & 0.882 \\
\hline$\sigma$ & 0.087 & 0.088 & 0.111 & 0.087 & 0.087 \\
\hline$h^{2}$ & 0.556 & 0.447 & 0.529 & 0.530 & 0.476 \\
\hline$\tau_{1}$ & 0.338 & 1.0 & - & 0.977 & 1.0 \\
\hline$\tau_{2}$ & 0.943 & 0.734 & - & 0.961 & 0.911 \\
\hline$\tau_{3}$ & 0.581 & 0.0 & - & 0.0 & 0.0 \\
\hline $\begin{array}{l}\chi^{2} \text { difference } \\
\text { general trans. } \\
\text { model (df) }\end{array}$ & - & $\begin{array}{l}4.8 \mathrm{NS}^{\mathrm{a}} \\
(2)\end{array}$ & $*$ & - & $\begin{array}{l}0.4 N^{a} \\
(2)\end{array}$ \\
\hline
\end{tabular}

${ }^{a}$ NS, not significant.

*22.2 (5 df) against the recessive general transmission model, $P<.001$.

$24.4(5 \mathrm{df})$ against the dominant general transmission model, $\mathrm{P}<.001$.

been reported elsewhere [Dadone et al, 1984; Turner et al, 1986]. After a power transformation to reduce residual skewness, there was still evidence for significant bimodality, although the estimates of the parameters of the distribution were altered so as to predict fewer observations in the upper component.

Before pooling the samples of pedigrees and blood bank donors for the segregation analyses, we found significant bimodality in each sample, and the parameter estimates were very similar to one another. The bimodality observed in the children suggests that the major effect contributing to the distribution of $\mathrm{Na}-\mathrm{Li}$ CNT is manifested at an early age. This is consistent with a hypothesis that the bimodality has a genetic etiology and inconsistent with the hypothesis that the bimodality may be a consequence of an environmental influence encountered by individuals as they age.

As expected, complex segregation analysis of the pooled sample also indicated that the data fit a mixture of two distributions. The general transmission model fit the data at least $10^{10}$ times better than the model with no major effect. Furthermore, these data reject the hypothesis that a major environmental effect explains the observed bimodality. The hypothesis that the effect that causes bimodality is transmitted from generation to generation is supported by the fit to these data of the restricted transmission model with a maximum likelihood estimate of $\tau_{2}=0.749$ for the recessive case and $\tau_{2}=0.869$ for the dominant case. The estimated standard deviation of the estimate of $\tau_{2}$ in the restricted transmission models was equal to 0.087 and 0.075 for the recessive and dominant cases, respectively. The maximum likelihood estimate of $\tau_{2}$ minus twice either of these standard deviations does not include the value of $\tau_{2}=$ 0.50 given by the Mendelian model.

Estimates of transmission probabilities that differ from those expected from well defined processes are difficult to interpret. $\tau_{2}$ may differ from the Mendelian expectation of 0.50 for any number of reasons, including alternative forms of genetic and environmental transmission or heterogeneous modes of transmission among subsets of the data. A subset of these data may fit a model of Mendelian transmission 
and a subset may fit another transmission model such that, when they are pooled, the best fitting model is given by the restricted transmission model. By evaluating the likelihood of each pedigree, we investigated whether a subset of families fit a model of Mendelian transmission better than others. Likelihoods were computed twice for each pedigree. They were computed once assuming that a mixed model including major gene and polygenic transmission is true, and once assuming that a model that includes a major environmental effect and polygenes explains the distribution of $\mathrm{Na}$ $\mathrm{Li}$ CNT values in the pedigrees. The estimates of the parameters that maximized the likelihood using all of the data (Table IV) were used in computing the likelihoods for individual pedigrees. The difference between the likelihoods for these two models is a measure of the relative support of the major gene as an explanation of the observed bimodality. There is statistically significant evidence $(\mathrm{P}<.05)$ for bimodality in the difference in $\log$ likelihoods between the major gene mixed model and the environmental mixed model for both the dominant and recessive cases. A fraction of the families give clear evidence for the segregation of a major locus effecting Na-Li CNT levels among family members. This analysis is consistent with the hypothesis that there is a heterogeneous etiology for the distribution of $\mathrm{Na}-\mathrm{Li} \mathrm{CNT}$ values in the population at large. However, the small size of the pedigrees prevents more detailed inferences.

The estimated correlations between relatives given in Table III are similar to those reported by others [Dadone et al, 1984; Lewitter and Canessa, 1985; Kagamimori et al, 1985]. These estimates are consistent with the estimates of the proportion of the phenotypic variance attributable to genetic differences among individuals obtained from the segregation analyses. Dadone et al [1984] analysed the distribution of Na-Li CNT values among members of ten Mormon pedigrees ascertained because of familial clustering of heart disease. Their data indicate that the observed distribution of $\mathrm{Na}-\mathrm{Li} \mathrm{CNT}$ values fit a mixture of two normal distributions better than a single normal distribution. Analyses of their data support the hypothesis that a major environmental factor is contributing to the observed bimodality. Application of a power transformation resulted in a single distribution that was not significantly skewed. For their transformed data, a model with both a major gene and polygenic inheritance did not fit the data statistically significantly better than a model of only polygenic inheritance. The discordances between their study and ours may be due to one or more differences between the two samples including the ascertainment scheme, the structure of the sample, and the population of inference.

Evidence is accumulating from the work presented here and that by others [Canessa et al, 1980; Boerwinkle et al, 1984; Dadone et al, 1984; Lewitter and Canessa, 1985; Kagamimori et al, 1985; Turner et al, 1986] supporting a genetic contribution to the variability in the distribution of Na-Li CNT values among individuals. This evidence includes (1) a larger correlation between first degree relatives than between genetically unrelated individuals; (2) an increased Na-Li CNT in normotensive offspring of essential hypertensives with higher Na-Li CNT levels but not in relatives of normotensive controls with lower Na-Li CNT levels; (3) a large polygenic contribution to the variability of $\mathrm{Na}-\mathrm{Li} \mathrm{CNT}$; (4) bimodality in the distribution of Na-Li CNT; (5) the absence of a description of an environmental factor or concomitant variable that accounts for the observed bimodality; and (6) the fact that, although not unequivocal, the data presented here support the hypothesis that the underlying discriminating factor responsible for the bimodality in at least a subset of the population may follow patterns best described by Mendelian transmission. 
There is general agreement that heredity plays an important role in the variability of blood pressure [Sing et al, 1985; Wiliams et al, 1984]. A phenotype as complex as blood pressure is not likely to have one genetic locus with a large effect, but rather is the result of the function and interaction of many genes and environments. No single genetic locus has yet been identified that is useful as a marker of hypertension or as a probe into the biological basis of interindividual blood pressure variability. We would like to assess what effect genetic variability for Na-Li CNT has on the distribution of blood pressure in the population. Using the results presented here and elsewhere, an ad hoc estimate of this effect can be made. Turner et al [1985] reported that $3 \%$ of the variability of systolic blood pressure can be accounted for by variability in Na-Li CNT among individuals. Under the polygenic model, about $50 \%$ of the NaLi CNT variability in this sample may be attributable to segregation of genetic factors. With assumptions, we may infer that $1.5 \%$ of the variability in blood pressure in the population is due to variability in loci affecting Na-Li CNT. Work is presently underway in our group to collect data on several hundred three-generation families and to estimate directly the contribution of the genetic effects on Na-Li CNT to the variability in blood pressure and to investigate further the possible genetic heterogeneity of this trait.

\section{ACKNOWLEDGMENTS}

The authors would like to thank Dr. P. Moll for her constructive comments concerning the analysis of this data and on this manuscript. This work has been supported in part by DOE contract DE-AC02-82ER60089 and NIH awards HL24489, HL30428, and 1-T32-GM-07544-05.

\section{REFERENCES}

Boerwinkle E, Turner ST, Sing CF (1984): The role of the genetics of sodium lithium countertransport in the determination of blood pressure variability in the population at large. In Brewer GJ (ed): "The Red Cell: Sixth Ann Arbor Conference." New York: Alan R. Liss, Inc., pp 479-507.

Boerwinkle E, Chakraborty R, Sing CF (1986): The use of measured genotype information in the analysis of quantitative phenotypes. I. Models and methods. Ann Hum Genet 50:181-194.

Blaustein MP (1984): Sodium transport and hypertension. Where are we going? Hypertension 6: 445-453.

Box GEP (1949): A general distribution theory for a class of likelihood criteria. Biometrika 36:317-346.

Canessa ML, Tosteson DC (1979): Determination of the sodium-lithium countertransport system of human erythrocytes. In Cooper TB, Gershon S, Kline NS, Schou M (eds): "Lithium: Controversies and Unresolved Issues." Amsterdam: Excerpta Medica, pp 978-982.

Canessa ML, Adragna NC, Solomon HS, Connolly TM, Tosteson BS, Tosteson DC (1980): Increased sodium-lithium countertransport in red cells of patients with essential hypertension. N Engl $\mathbf{J}$ Med 302:772-776.

Cannings C, Thompson EA, Skolnick MH (1978): Probability functions on complex pedigrees. Adv Appl Prob 10:26-61.

Dadone MM, Hasstedt SJ, Hunt SC, Smith JB, Ash KO, Williams RR (1984): Genetic analysis of sodium-lithium countertransport in 10 hypertension prone kindreds. Am J Med Genet 17: $565-577$.

Day NE (1969): Estimating the components of a mixture of normal distributions. Biometrika 56: $463-479$.

Demenais F, Lathrop M, Lalouel JM (1986): Robustness and power of the unified model in the analysis of quantitative measurements. Am J Hum Genet 38:228-234. 
Elston RC, Stewart J (1971): A general model for the genetic analysis of pedigree data. Hum Hered $21: 523-542$.

Hasstedt SJ (1982): A mixed model likelihood approximation on large pedigrees. Comput Biomed Res 15:295-307.

Hasstedt SJ, Cartwright PE (1981): "PAP: Pedigree Analysis Package, Rev. 2. Technical Report No. 13." Salt Lake City: Department of Medical Biophysics and Computing, University of Utah.

Kagamimori S, Naruse Y, Takata M, Fujita T, Watanabe M (1985): Familial aggregation of red blood cell cation transport systems in Japanese families. Am J Epid 122:386-390.

Keith RA, Van Loon J, Wussow LF, Weinshilboum RM (1983): Thiol methylation pharmacogenetics: Heritability of human erythrocyte thiol methyltransferase activity. Clin Pharmacol Ther 34: $521-528$.

Lalouel JM (1979): GEMINI-A computer program for optimization of general nonlinear functions. Technical Report No. 14. Salt Lake City; Department of Medical Biophysics and Computing, University of Utah.

Lalouel JM, Rao DC, Morton NE, Elston RC (1983): A unified model for complex segregation analysis. Am J Hum Genet 35:816-826.

Lewitter FI, Canessa M (1985): Red cell sodium transport studies in adult twins. Am J Hum Genet suppl 36:172s.

MacLean CJ, Morton NE, Elston RC, Yee S (1976): Skewness in commingled distributions. Biometrics 32:695-699.

Sing CF, Boerwinkle E (1985): The genetics of blood pressure variability: An overview. In Filer LJ, Jr, Lauer RM (eds): "Children's Blood Pressure." Report of the Eighty-Eighth Ross Conference on Pediatric Research, Columbus, OH: Ross Laboratories, pp 35-43.

Swales JD (1983): Abnormal ion transport by cell membranes in hypertension. In Robertson JIS (ed): "Handbook of Hypertension: Clinical Aspects of Essential Hypertension." Amsterdam: Elsevier Science Publishers BV Vol. 1 pp 239-266.

Trevisan M, Ostrow D, Cooper R, Liu K, Sparks S, Stamler J (1981): Methodological assessment of assays for red cell sodium concentration and sodium-dependent lithium efflux. Clin Chim Acta 116:319-329.

Turner ST, Johnson M, Boerwinkle E, Richelson E, Taswell HF, Sing CF (1985): Distribution of sodium-lithium countertransport and its relationship to blood pressure in a large sample of blood donors. Hypertension 7:955-962.

Turner ST, Boerwinkle E, Johnson M, Richelson E, Sing CF (1986): Sodium-lithium countertransport in erythrocytes of ambulatory hypertensive and normotensive patients. Hypertension (submitted).

Weder AB (1985): Red-cell lithium-sodium countertransport and renal lithium clearance in hypertension. N Engl J Med 314:198-201.

Weinshilboum RM (1979): Hypertension: A biochemical genetic approach. In Sing CF, Skolnick M (eds): "The Genetic Analysis of Common Diseases: Application to Predictive Factors in Coronary Disease." New York: Alan R. Liss, Inc., pp 157-181.

Williams RR, Dadone MM, Hunt SC, et al (1984): The genetic epidemiology of hypertension: A review of past studies and current results for 948 persons in 48 Utah pedigrees. In Rao DC, Elston RC, Kuller LH, Feinleib M, Carter C, Havlik R (eds): "Genetic Epidemiology of Coronary Heart Disease: Past, Present, and Future.” New York: Alan R. Liss Inc, pp 419-442.

Wolstenholm G, Cameron M (1954): "CIBA Foundation Symposium on Hypertension." Little Brown and $\mathrm{Co}$.

Edited by D.C. Rao 